\title{
2 Die Waffen-SS: Ideen, Ideologien und Umsetzung
}

Im folgenden Kapitel soll ein Überblick darüber gegeben werden, auf welchen Ideen und Ideologien die Waffen-SS basierte und wie ihre Umsetzung aussah. Es sollen dabei die wichtigsten Punkte im Hinblick auf die Rekrutierungen in Südosteuropa vorgestellt werden. Natürlich können in einer solchen Überblicksdarstellung viele Aspekte nur skizziert und nicht näher beleuchtet werden.

Nach Hitlers Machtantritt im Januar 1933 begannen sich nicht nur die Lebenswelten der Deutschen radikal zu verändern. Im Schatten der damals wesentlich einflussreicheren und mächtigeren, parteieigenen Sturmabteilung (SA) wuchs eine kleine, zum Schutze Hitlers aufgestellte, Organisation heran. ${ }^{1}$ Die Schutzstaffel (SS) war aber als neuartige Institution innerhalb des nationalsozialistischen Einflussbereichs in ihren ersten Jahren „nur ein kleines, zunächst wenig bedeutsames soziales Subsystem“, wie Wegner sie beschreibt, dessen Position und Funktion innerhalb des NS-Systems umstritten war und dessen Überleben weitgehend davon abhing, wie stark der spätere Reichsführer SS Heinrich Himmler seinen persönlichen Machtbereich ausbauen konnte. ${ }^{2}$ Nach dem RöhmPutsch Ende Juni 1934 und der damit einhergehenden Ermordung der SA-Führung war der Grundstein gelegt für den Erfolg der SS als durchschlagende Parallelinstitution zu herkömmlichen Organen wie der Polizei und der Wehrmacht. ${ }^{3}$

\subsection{Organisation und Ausbau: Der Weg zur staatlichen Institution}

Am 4. April 1925 von Hitler als seine persönliche Leibgarde ins Leben gerufen, wurde die SS in den ersten Jahren vor allem mit polizeilichen Aufgaben betraut. Seit 1926 war sie der SA unterstellt. ${ }^{4} 1929$ bestanden bereits 22 Standarten. Eine Standarte umfasste 1.000 bis 3.000 Mann. Der zukünftige Reichsführer SS Heinrich Himmler war im selben Jahr zum Chef der SS, damals noch der SA un-

\footnotetext{
1 Zur Geschichte der SA vgl. Daniel Siemens, Stormtroopers. A New History of Hitler's Brownshirts, New Haven etc.: Yale University Press 2017.

2 Wegner, Soldaten, S. 181. Vgl. auch Rohrkamp, Weltanschaulich gefestigte Kämpfer, S. 158.

3 Vgl. Martin Broszat, Nationalsozialistische Konzentrationslager 1933-1945, in: Hans Buchheim et al., Anatomie des SS-Staates, Bd. 2: Konzentrationslager, Kommissarbefehl, Judenverfolgung, Olten/Freiburg i. Br. $1984^{4}$, S. 38.
}

4 Vgl. Siemens, Stormtroopers, S. 33 f.

Ә Open Access. ( 2021 Franziska Anna Zaugg, publiziert von De Gruyter. (c) BY Dieses Werk ist 
terstellt, ernannt worden und hatte auf 12 dieser Standarten direkten Zugriff. ${ }^{5}$ Aufgrund von Aufstiegsmöglichkeiten auch für Männer aus den unteren sozialen Schichten wuchs die SS in ihren Anfangsjahren rasch: 1929 war sie noch eine kleine Organisation von 250 Mann, 1930 verfügte sie über 2.000 und 1931 bereits über 10.000 Mann. ${ }^{6}$

Von 1933 bis 1939 baute die SS unter der Führung Himmlers ihre Einflusssphäre stetig aus. In diesem Zeitraum entstanden neben der Allgemeinen SS drei bewaffnete Formationen, die schließlich 1939 in der Waffen-SS aufgehen sollten, aber vorerst noch ohne spezifisch militärischen Auftrag blieben. ${ }^{7}$ Bereits im März 1933 war die „Leibstandarte Adolf Hitler“ (LSSAH) aufgestellt worden. Im September 1934 folgte die „SS-Verfügungstruppe“ (SS-VT), kurz darauf die „SS-Totenkopfverbände“ (SS-TV). Neben ihrer ursprünglichen Aufgabe als Eingreiftruppe der Partei wurden die bewaffneten Verbände, insbesondere die Totenkopfverbände, für die Bewachung der Konzentrationslager (KZ) eingesetzt.

Bemerkenswert an dieser Phase ist, dass sich die SS einerseits aus dem Korsett der Rechtstaatlichkeit zu lösen vermochte, gleichzeitig aber vermehrt gerade als staatliche Instanz im NS-System auftrat und somit die von Himmler gewünschte Nähe zu anderen Institutionen des Dritten Reiches fand. Während die Allgemeine SS auf Vereinsbasis weiterexistierte, entwickelte sich die bewaffnete SS zur paramilitärischen Organisation: Die Ernennung Himmlers zum Chef der deutschen Polizei in Personalunion und die damit verbundene Verzahnung von SS und Polizei ebnete dem bewaffneten Flügel den Weg, um ,an dem seit 1934 rapide zunehmenden politischen Eigengewicht der Gesamtorganisation angemessen zu partizipieren“ und bis 1938 „eine wehrpolitische Bedeutung [zu erreichen], die [ihr] Verhältnis zum traditionellen Waffenträger der Nation [Reichswehr/Wehrmacht] auf eine neue Grundlage stellen sollte. “8 Die bewaffneten Verbände der SS waren somit in ein Konkurrenzverhältnis zur Wehrmacht getreten und mussten gegenüber dieser traditionellen staatlichen Organisation neu erkämpfte Kompetenzbereiche definieren und verteidigen. ${ }^{9}$ Die Vertreter der Wehrmacht reagierten mit Kritik am bewaffneten Flügel der SS und bezeichneten seine Mitglieder als minderwertig. ${ }^{10}$

5 Wegner, Anmerkungen zur Geschichte der Waffen-SS, S. 407.

6 Kogon, SS-Staat, S. 23.

7 Wegner, Anmerkungen zur Geschichte der Waffen-SS, S. 407.

8 Wegner, Soldaten, S. 95.

9 Selbst innerhalb des „schwarzen Ordens“ kam es zu Rivalitäten mit anderen Teil-Institutionen der SS. Vgl. Leleu, La Waffen-SS, S. 392.

10 Dass sich die sozialen Milieus von Anwärtern für Wehrmacht- und SS-Karrieren wirklich unterschieden, zeigen beispielsweise die Studien von Wegner und Rohrkamp. Vgl. Rohrkamp, 
Die SS war auf dem Weg, im Dritten Reich genauso Teil staatlicher Infrastruktur zu werden wie etwa das Auswärtige Amt. Zwei Zäsuren lassen die Grundsteinlegung für die Entwicklung der SS zur staatlichen Institution deutlich werden: Erstens das preußische Gesetz über die Geheime Staatspolizei (Gestapo) vom 10. Februar 1936, zweitens die Ernennung Himmlers zum Chef der deutschen Polizei am 17. Juni 1936 - zusätzlich zu seiner Funktion als Reichsführer SS. Dies hatte zur Folge, dass nun die Bereiche von Polizei und politischer Polizei endgültig ineinander übergingen. ${ }^{11}$ Das durch Kogon und die Nürnberger Prozesse geprägte Bild vom „Staat im Staate“, ${ }^{12}$ welches die Forschung während Jahrzehnten dominierte, gilt daher heute als überholt. ${ }^{13}$ Die SS muss als Teil des nationalsozialistischen Staates gesehen werden, dessen Ämterdarwinismus auf der einen, die Zusammenarbeit zwischen den einzelnen Stellen auf der andere Seite, Macht und Alltag der Institution bestimmte. ${ }^{14}$ Mochte gegen außen lange Zeit der Anschein einer straffen Durchstrukturiertheit vorherrschen; ${ }^{15}$ im Innern war der nationalsozialistische Verwaltungsapparat - so auch die SS - geprägt von Ad-hoc-Entscheiden, Interpretationsdifferenzen, von Kompetenzstreitigkeiten und dem Machtstreben Einzelner. ${ }^{16}$

Zwischen 1935 und 1938 verfünffachte sich die Anzahl der Angehörigen der „Totenkopfverbände“, die Verfügungstruppe wuchs um das Dreifache: Diese starke Wachstumsphase in der Vorkriegszeit wie auch die standartenweise Zusammenfassung genannter Verbände wurden angesichts der gleichzeitig ablaufenden und wesentlich größer angelegten Umstrukturierung der Wehrmacht

Weltanschaulich gefestigte Kämpfer, S. 64-77 und 562. Vgl. Wegner, Soldaten, Kapitel „Soziale Herkunft und beruflicher Werdegang“, S. 217-242. Siehe zur Positionierung der Waffen-SS im NS-Staat Leleu, La Waffen-SS, S. 21-24, 115f., 123-125.

11 Vgl. Hans Buchheim, Anatomie des SS-Staates, 1. Bd., Freiburg i. Br. u. a.: Walter-Verlag $1984^{4}$, S. 59.

12 Der Begriff wurde von Eugen Kogon bereits 1946 geprägt. Vgl. Eugen Kogon, Der SS-Staat, S. $20 \mathrm{f}$.

13 Vgl. Schulte, SS, Himmler und die Wewelsburg, S. XIII-XIV.

14 Siehe dazu beispielsweise Johannes Houwink ten Cate, Gerhard Otto (Hgg.), Das organisierte Chaos: „Ämterdarwinismus“ und „Gesinnungsethik“: Determinanten nationalsozialistischer Besatzungsherrschaft, Berlin: Metropol 1999. Christian Gerlach, Rivalry and Competition, in: Wolfgang Seibel/Gerald D. Feldman (Hgg.), Networks of Nazi Persecution: Bureaucracy, Business and the Organization of the Holocaust, New York and Oxford: Berghahn 2004, S. $13 \mathrm{f}$.

15 Vgl. etwa Oliver Lubrich, Reisen ins Reich 1933-1945. Ausländische Autoren berichten aus Deutschland, Frankfurt a. M.: Eichborn 2004, bspw. Denis de Rougemont, in: ebd., S. 109-113. 16 Vgl. zur „Ausdehnung des innerdeutschen Machtkampfes auf die besetzten Gebiete“ Umbreit, Zur Organisation der Besatzungsherrschacft, in: Houwink ten Cate, Otto (Hgg.), Das organisierte Chaos, S. 49-51. 
lange unterschätzt. ${ }^{17}$ Trotz des rasanten Wachstums der bewaffneten SS herrschte weder auf der Ebene des höheren Offizierkorps der Wehrmacht, dessen Angehörige die bloße Existenz einer bewaffneten Parallelorganisation als Misstrauensbeweis werteten, noch bei Himmler selbst, der den Anspruch hatte, seine SS für die Kriegführung gegen Außen als auch im Innern einzusetzen, das Verlangen, sich einander anzunähern. Und dies, obwohl die Waffen-SS hinsichtlich der militärischen Ausbildungslehrgänge auf die Wehrmacht angewiesen war. ${ }^{18}$ Hitlers Erlass vom 17. August 1938 ebnete schließlich das letzte Wegstück, damit die Waffen-SS einerseits unter dem Befehl des Reichsführer SS und Chef der Deutschen Polizei, Himmler, gegen den inneren Feind, andererseits im Kriegsfall unter dem Oberbefehl der Wehrmacht gleichberechtigt neben dieser eingesetzt werden konnte. ${ }^{19}$

\subsection{Das Ideal der SS als Elite-Orden und die „weltanschauliche Schulung" als seine Grundlage}

Neben der Funktion der Schutzstaffel zum persönlichen Schutz des „Führers“, verstanden sich ihre Mitglieder als Schlüsselorganisation zur Umsetzung des Nationalsozialismus und als Garant für das Fortbestehen und den Ausbau nationalsozialistischer Herrschaft. ${ }^{20}$ Die SS wurde daher von Beginn an als Institution konzipiert, deren Ordenscharakter für ihre Angehörigen Ausgangs- und Rückbezugspunkt sein sollte: ${ }^{21}$ Sie sollte eine Organisation sein, deren Mitglieder sich durch ihre Zugehörigkeit von den übrigen Bürgern des Dritten Reiches abheben sollten. ${ }^{22}$ Die eigenen Gesetze und Rituale, welche die Basis der SS bildeten, bauten auf zahlreichen historischen Vorgängerinstitutionen auf, von welchen sich Himmler Elemente für seinen „Orden“ borgte; so etwa der Jesuitenorden oder der Deutsche Ritterorden. ${ }^{23}$ Indem Himmler die SS als Orden konzipierte, konnte er sie auch als Religionsalternative präsentieren. Dadurch beabsichtigte er längerfristig, den Einfluss der beiden großen Landeskirchen eindämmen, die teilweise

17 Vgl. Wegner, Soldaten, S. 103.

18 Vgl. ebd., S. 113f. Vgl. Jürgen Förster, Was wäre die Waffen-SS ohne den Generalstab gewesen? Zur strukturellen Zusammenarbeit von Heer und SS, in: Michael Jonas u. a. (Hg.): Dynamiken der Gewalt. Krieg im Spannungsfeld von Politik, Ideologie und Gesellschaft, Paderborn 2015, S. 216-222.

19 Vgl. Wegner, Soldaten, S. 115.

20 Vgl. Kogon, SS-Staat, S. 23.

21 Vgl. ebd., S. 22.

22 Vgl. Peter Longerich, Himmler, S. 314 f., 365.

23 Vgl. Wegner, Soldaten, S. 38-41. Longerich, Himmler, S. 297-304. 
auf ihre Mitglieder noch immer einen starken Einfluss ausübten - auch hinsichtlich ethischer Grundhaltungen. ${ }^{24}$

Nicht intellektuelle, sondern vor allem „rassische“ bzw. körperliche Merkmale bildeten die Grundlage für die „Auslese der Besten“: ${ }^{25}$ Nach seiner Ernennung zum „Reichsführer SS“ im Januar 1929 begann Heinrich Himmler damit, die Auswahlkriterien bei der Rekrutierung für die Waffen-SS zu konkretisieren. Ein Befehl vom 4. Oktober 1932 legte fest, dass nur unter ärztlicher Aufsicht als tauglich befundene, höchstens 30 Jahre alte, mindestens 1.70 Meter große und „rassisch hervorragende“ Männer zu rekrutieren seien. Für die Aufnahme in die „Verfügungstruppe“ galten sogar noch strengere Kriterien: Das Höchstalter betrug 23 Jahre, die Mindestgröße 1.74 Meter. Brillenträger waren in der Regel nicht zugelassen - dies obwohl der prominenteste SS-Mann selbst ein Brillenträger war. ${ }^{26}$ Da ein Bewerber für die Führerlaufbahn weder das Abitur noch einen anderen qualifizierenden Schulabschluss nachweisen musste, stieß die Werbung bei gebildeteren Teilen der männlichen jugendlichen Bevölkerung auf Zurückhaltung; bisher aufgrund ihrer Herkunft oder Ausbildung weniger Qualifizierten bot die SS neue Aufstiegsmöglichkeiten. ${ }^{27}$ Obwohl die Führerlaufbahn aufgrund dieser Neuerung insbesondere für Bewerber aus der unteren Mittelschicht attraktiv wirkte, ${ }^{28}$ waren diese vor allem im Bereich der unteren Führungsfunktionen zu finden. Bei höheren Dienstgraden sah die Zusammensetzung anders aus. Sie rekrutierten sich, nicht zuletzt aufgrund ihrer Berufserfahrung, zu großen Teilen aus Heeres- und Polizeioffizieren, ${ }^{29}$ also aus denselben Schichten, die auch im Heer traditionellerweise bessere Aufstiegschancen hatten. Dies führte dazu, dass die SS bereits in früheren Jahren, lange vor den Rekrutierungen in Südosteuropa, ein sehr heterogenes Konstrukt bildete, dessen inneres Konfliktpotential von Beginn an hoch war. ${ }^{30}$

Als wichtigstes Instrument zur Umsetzung der Ordensidee galt die ideologische Schulung, die unter dem Begriff der „weltanschaulichen Erziehung“ zusammengefasst dem Amt IV des SS-Hauptamtes und somit Gottlob Berger

24 Vgl. bspw. Hubert Gruber, Katholische Kirche und Nationalsozialismus, 1930-1945, Paderborn: Schöningh 2006, S. 237-239, 258, 358. Vgl. ebenfalls Longerich, Himmler, S. 298 f.

25 Vgl. Wegner, Soldaten, S. $135 \mathrm{f}$.

26 Vgl. ebd., S. 135. Vgl. zu den Ausnahmen etwa Rudolf von Ribbentrop, Sohn von Reichsaußenminister Joachim von Ribbentrop, als Brillenträger bei der Waffen-SS. Rudolf von Ribbentrop, Mein Vater Joachim von Ribbentrop. Erlebnisse und Erinnerungen. Graz: Ares 2008, S. 364.

27 Vgl. Wegner, Soldaten, S. 140, 143.

28 Vgl. Rohrkamp, Weltanschaulich gefestigte Kämpfer, S. 64-69 und S. 562. Vgl. Wegner, Soldaten, S. 335.

29 Vgl. ebd., S. 285.

30 Vgl. ebd., S. 335. 
unterstand. ${ }^{31}$ Die „weltanschauliche Schulung“ sollte wesentliche Werte des Nationalsozialismus im Allgemeinen und der SS im Besonderen vermitteln. ${ }^{32}$ Andererseits sollten dadurch auch die in Bezug auf Qualifikation, soziale Herkunft und Ausbildung großen Unterschiede innerhalb der Truppe nivelliert und auf ein gemeinsames Niveau gebracht werden. ${ }^{33}$ In den Junkerschulen für die SS-Führerausbildung wurde der ideologischen Erziehung besondere Aufmerksamkeit geschenkt. Denn diese Institution hatte nicht nur einen Bildungs- bzw. Ausbildungsauftrag, sondern auch eine erzieherische Funktion. ${ }^{34}$ Hauptinhalte waren etwa die „ewigen Lebensgesetze“, ihre Verwirklichung im Nationalsozialismus und die Darlegung der deutschen und europäischen Geschichte unter einem rassengeschichtlichen und geopolitischen Blickwinkel. All diese Lerninhalte basierten auf dem Leitgedanken eines Führungsanspruchs Deutschlands. ${ }^{35}$ Die Vermittlung von solchem „Orientierungswissen“ sollten dem jungen SS-Führer helfen, immer eine Erklärung bereit $\mathrm{zu}$ haben und nie um eine Antwort verlegen zu sein. ${ }^{36}$

Neben einer fundierten nationalsozialistischen Schulung durch „weltanschauliche Erziehung“ sollten die Führeranwärter in den Junkerschulen der SS eine traditionelle militärische Ausbildung ähnlich derjenigen in der Wehrmacht erhalten. Die Institutionalisierung von nationalsozialistischem Ethos und militärischem Professionalismus diente als Grundlage für die Schaffung dieser neuartigen Elite: „Eine Elite, die militärisch sein konnte, ohne ,das Militär‘ zu sein, und die nationalsozialistisch sein konnte, ohne, die Partei‘ zu sein“, wie Wegner diesen Anspruch definiert. ${ }^{37}$ Neuartig und ein Unterschied zur Wehrmacht war, dass die Ausbildung keine Trennung zwischen zukünftigen Führungsfunktionen eines Truppenoffiziers und der Verwaltungstätigkeit eines Beamten vorsah. ${ }^{38}$

31 Vgl. Rohrkamp, Weltanschaulich gefestigte Kämpfer, S. 437.

32 Vgl. Leleu, La Waffen-SS, S. 413-470. Vgl. zur Entwicklung der „weltanschaulichen Schulung “ auch Harten, Himmlers Lehrer. Die weltanschauliche Schulung in der SS 1933-1945, Paderborn: Ferdinand Schöningh 2014, S. $41 \mathrm{f}$.

33 Vgl. Wegner, Soldaten, S. 149, 185.

34 Wegner, Soldaten, S. 158. Vgl. Harten, Himmlers Lehrer, S. 303.

35 Wegner, Soldaten, S. 164.

36 Ebd., S. 165.

37 Vgl. Wegner, Soldaten, S. 171.

38 Vgl. Wegner, Soldaten, S. 147. Diese Aufhebung der Unterschiede in der Ausbildung von Truppenoffizieren und Verwaltungsbeamten sollte sich in den letzten Kriegsmonaten und der prekären personellen Situation etwa in Albanien und im Kosovo als Vorteil erweisen; Verwaltungsführer wurden kurzerhand als Ausbilder in der neu aufgestellten Division "Skanderbeg" eingesetzt. Vgl. Brief von Pistor an Vopersal, undatiert, BArchF, N 756/182b. 
Seit Mitte 1938 fanden für Führeranwärter sechs- bis achtwöchige Vorbereitungslehrgänge statt, die eine Standardisierung der Ausbildung gewährleisten sollten. Trotz der Vereinheitlichung der Ausbildungslehrgänge blieb aber die Diskrepanz zwischen dem von Himmler angestrebten Ideal und dem realen Qualifikationsniveau der Truppe bestehen. Detaillierte Pläne, wie die ideologische Ausbildung ablaufen und welche Stoffeinheiten gelernt werden sollte, nützten hinsichtlich der Tatsache, dass qualifiziertes Ausbildungspersonal fehlte, wenig. ${ }^{39}$ Die Schwierigkeit, „weltanschauliche Erziehung“ an den Mann zu bringen, sollte sich im Laufe des Krieges und mit der Ausdehnung auf „fremdvölkische“ Bewerber noch verschärfen. ${ }^{40}$ Auch die drei Grundpfeiler der SS, „Rasse“, „Freiwilligkeit“ und ideologischer Fanatismus, ${ }^{41}$ gerieten ins Wanken und mussten später, bei der Rekrutierung von „Fremdvölkischen“, aufgrund von Sachzwängen ganz aufgegeben werden. ${ }^{42}$

Schließlich unterschied sich die Ausbildung der SS-Führerbewerber an den Junkerschulen kaum von den im Heer üblichen Offiziers-Lehrgängen. Sie verlief weitestgehend in Anlehnung an die im Heer geltenden Dienstvorschriften. ${ }^{43}$ Die front- und truppendienstbezogenen Lehrfächer wie Taktik, Geländekunde, Heerwesen oder Waffenlehre nahmen nach Kriegsausbruch gegenüber den nicht direkt für den Kriegsdienst relevanten Lehrfächern wie „weltanschauliche Erziehung“ noch an Wichtigkeit zu. ${ }^{44}$ Nebst der „weltanschaulichen Erziehung“ wurde allerdings auch im Fach „Heerwesen“ Wert auf die Vermittlung SS-spezifischer Führungs- und Verhaltensgrundsätze gelegt: ${ }^{45}$

Im Mittelpunkt des Unterrichts stand darum insbesondere die Vermittlung eines SS-gemäßen Führerleitbildes und die Einübung eines einheitlichen Beurteilungs- und Entschei-

39 Vgl. Wegner, Soldaten, S. 156 f. Vgl. Rohrkamp, Weltanschaulich gefestigte Kämpfer, S. 147. 40 Vgl. Jürgen Förster, Die weltanschauliche Erziehung in der Waffen-SS. „Kein totes Wissen, sondern lebendiger Nationalsozialismus“, in: Jürgen Matthäus, Konrad Kwiet, Jürgen Förster, Richard Breitmann, Ausbildungsziel Judenmord? „Weltanschauliche Erziehung“von SS, Polizei, Waffen-SS im Rahmen der „Endlösung“, Frankfurt a.M.: Fischer 2003, S. 93-99, 107-113. Vgl. hierzu auch die beiden Kapitel „Die Integration ,Volksdeutscher““ und „Die Integration ,Fremdvölkischer““ in der vorliegenden Studie, S. 41-45.

41 Rohrkamp, Weltanschaulich gefestigte Kämpfer, S. 57.

42 Vgl. Birn, SS - Ideologie und Herrschaftsausübung, S. 74.

43 Wegner, Soldaten, S. 159, 162. Allerdings ergänzt Wegner hier, diese Beurteilung stehe unter dem Vorbehalt, dass eine detaillierte militärfachliche Analyse des Ausbildungsmaterials der Junkerschulen noch ausstehe.

44 Vgl. ebd., S. 161. Himmler betonte indes immer wieder, dass die weltanschauliche Erziehung an den Junkerschulen der militärischen Ausbildung gleichgestellt sei. Wegner, Soldaten, S. 168.

45 Vgl. zur Aufhebung der Trennung „weltanschaulicher Erziehung“ und militärischer Ausbildung auch Förster, Die weltanschauliche Erziehung, S. 94. 
dungsverhaltens, vor allem im Hinblick auf den Umgang mit Untergebenen. Aber auch Vorschriften etwa des Kriegsvölkerrechts gehörten zum Themenspektrum des Faches, ebenso Verhaltenkodices für nichtmilitärische Situationen wie ,Grundgesetze' der SS oder Fragen der gesellschaftlichen Etikette. Mithin wird offensichtlich, dass das Fach ,Heerwesen' in hohem Maße Teil einer fächerübergreifenden Persönlichkeitserziehung war. Es war der Versuch, allgemeine militärische wie auch SS-ideologische Normen zu operationalisieren und sie für den täglichen Truppen- und Lebensalltag verwertbar zu machen. ${ }^{46}$

Nach Ausbruch des Kriegs sollte die schwieriger werdende Abgrenzung zwischen Waffen-SS und Wehrmacht durch die der Konzeption der Waffen-SS als politisch-militärische Organisation zugrunde liegende „weltanschauliche Schulung“ wieder klar definiert werden, wie Leleu schreibt. ${ }^{47}$ Um diesem politisch-militärischen Doppelanspruch zu genügen, ${ }^{48}$ sollte „weltanschauliche Erziehung“ nicht bloss eine „wehrgeistige Erziehung“ liefern, wie sie die Wehrmacht anbot, sondern eine „weltanschauliche“ Bildung liefern, die darüber hinausging. ${ }^{49}$ Der Führer der Abteilung VI der Division „Handschar“, SS-Hauptsturmführer Ekkehard Wangemann, schreibt zu dieser Abgrenzung: „es ist keineswegs zu vergessen, dass die Wehrmacht [...] sich mit Politik nie befasst hat, ja, dass vor 10 Jahren deutschen Soldaten noch nicht einmal das Wahlrecht zustand, jedoch hat diese [nationalsozialistische] Erziehung ihre besondere Ursache und der Totalitätsanspruch einer großen politischen, weltanschaulichen Idee, [sic] dürfte einmalig sein.“50

Im Krieg wurde die Koordination der politischen Erziehungsarbeit „Weltanschauliche Erziehung“ durch den „Weltanschauliche Erziehungs-Führer“, den Führer der Abteilung VI, beibehalten, allerdings die Vermittlung der „Weltanschaulichen Schulung“ im Wesentlichen Aufgabe des Kompaniechefs und eingeschränkt des Bataillonskommandeurs. ${ }^{51}$ Letztlich war diese Neuerung nicht praktischer bzw. führungstechnischer Natur, sondern auch eine logische Konsequenz eines Gesamtkonzepts, das den SS-Mann als eine „Verschmelzung von militärischem Können und weltanschaulichem Aktivismus“sah, der so als

46 Wegner, Soldaten, S. 164.

47 Vgl. Leleu, Jenseits der Grenzen, S. $37 \mathrm{f}$.

48 Vgl. Wegner, Soldaten, S. 201.

49 Rohrkamp, Weltanschaulich gefestigte Kämpfer, S. 437.

50 Gedächtnisaufzeichnung der „Dienstbesprechung der Kommandeure und Imame“ Führer der Abteilung VI, SS-Hauptsturmführer Ekkehard Wangemann, 8.4.1944, BArchB, NS 19/2601, Bl. 186. Dass die Wehrmacht durchaus einer Indoktrination unterzogen wurde, bewies Manfred Messerschmidt schon früh in seiner Studie Die Wehrmacht im NS-Staat. Zeit der Indoktrination, Hamburg: Decker 1969. Siehe hierzu auch Jürgen Förster, Die Wehrmacht im NS-Staat: Eine strukturgeschichtliche Analyse, München: R. Oldenbourg 2007.

51 Diese Aufgabenteilung bestand bereits seit 1937. Vgl. Wegner, Soldaten, S. 189. 
Verkörperung des politischen Soldaten seine Untergebenen lehren und ihnen gleichzeitig Vorbild in militärischer wie auch weltanschaulicher Hinsicht zu sein hatte. ${ }^{52}$ Für SS-Hauptsturmführer Wangemann, Führer der Abteilung VI der Division „Handschar“, ähnelte seine Funktion der eines „Politruks“, des Politkommissars bei der Roten Armee, der als „Gehilfe des Div[isions]kommandeurs in weltanschaulichen und politischen Fragen“ eingesetzt sei. ${ }^{53}$

Nach Hitlers Feldzügen 1940 im Westen und Norden wurde folgerichtig auch das weltanschauliche Schulungsmaterial in andere Sprachen, etwa Holländisch oder Flämisch, übersetzt. ${ }^{54}$ Seit 1941 war die „Germanische Leitstelle“, welche die Koordination der „germanischen“ Freiwilligenwerbung übernommen hatte, nicht nur für die Anwerbung und Ergänzung, sondern auch für die Schulung der Freiwilligen aus anderen Ländern zuständig. ${ }^{55}$ Für die außerhalb Deutschlands aufgestellten Verbände war die Abteilung 1.1f „Europäische Erziehungsarbeit“, Amtsgruppe C I des SS-Hauptamtes zuständig, die bis August 1943 Hans Lüdemann, später Erich Kruschinsky leitete. Der Bedarf an länderspezifischen Schulungsunterlagen stieg durch die neuen ost- und südosteuropäischen Verbände stark an. Diese sollten vor allem auf die „gesamteuropäische Legitimation“ der Waffen-SS ausgerichtet sein. An Wichtigkeit gewannen auch Richtlinien und Anweisungen für den Umgang mit und die Ausbildung von „fremdvölkischen“ Einheiten, die Unterführer und Führer auf den Umgang mit diesen „neuartigen“ Freiwilligen vorbereiten sollten. ${ }^{56}$

Nach dem Balkanfeldzug 1941 und verstärkt seit 1942 wurden in großer Zahl „Volksdeutsche“, sowie seit 1943 bosnische und schließlich 1943/44 albanische Muslime und Katholiken geworben und auch im Osten Europas schritten

52 La Waffen-SS, S. Wegner, Soldaten, S. 188-190. Vgl. zur Umsetzung dieser Aufgabe durch Vorgesetzte bei Leleu, der einleuchtend darstellt, dass die praktische Umsetzung vielfach zu wünschen übrig ließ. Vgl. Leleu, La Waffen-SS, S. 426.

53 Gedächtnisaufzeichnung der „Dienstbesprechung der Kommandeure und Imame“ Führer der Abteilung VI, SS-Hauptsturmführer Ekkehard Wangemann, 8.4.1944, BArchB, NS 19/2601, Bl. 187. Allerdings existierte im Dritten Reich seit Ende 1943 auch innerhalb der Wehrmacht die Funktion des sogenannten nationalsozialistischen Führungsoffiziers, der für die weltanschauliche Erziehung der Truppe zuständig war. Vgl. Waldemar Besson, Zur Geschichte des nationalsozialistischen Führungsoffiziers, in: Vierteljahrshefte für Zeitgeschichte 1 (1961), S. 76-116. Vgl. auch Leleu, Jenseits der Grenzen, S. 40. Vgl. zum Vorbild der politischen Kommissare in der Roten Armee in der Schulung der SS auch Wegner, Soldaten, S. 188.

54 Vgl. Hans-Christian Harten, Himmlers Lehrer, S. 351-355.

55 Vgl. Harten, Himmlers Lehrer, S. 355. Ausführlich zur „Germanischen Leitstelle“ siehe Harten, Himmlers Lehrer, S. 355-368.

56 Vgl. Harten, Himmlers Lehrer, S. 409-411. Vgl. hierzu auch erhellend Kaesdorfs Anleitung, "Erfahrungen mit albanischen Soldaten”, undatiert, BArchB, NS 31/444, Bl. $23-28$ und „Der Deutsche in der Sicht des Südostens“, undatiert, BArchB, NS31/422, Bl. 19-28. 
die Rekrutierungen voran. ${ }^{57}$ Schon 1942 bemerkte SS-Standartenführer Rudolf Brandt in einem Austausch der SS mit dem Oberkommando der Wehrmacht (OKW), dass in den Reihen der „Volksdeutschen“ der Division „Prinz Eugen“ dringend „weltanschauliche“ und politische Erziehung notwendig sei. ${ }^{58}$ Auch für die Division „Handschar“ lässt sich die Konzeption „,weltanschaulicher Schulung“ noch 1944 von Seiten der Deutschen, aber auch in Reden des Großmufti von Jerusalem nachweisen. Die Umsetzung der „weltanschaulichen“ Schulung sollte in dieser Division sogar auf zwei Ebenen erfolgen, über die Kompanieführer und die Imame. ${ }^{59}$

„Weltanschauliche Erziehung“ war als fächerübergreifendes pädagogisches Prinzip zu verstehen, mit dem Ziel, die Persönlichkeit der Soldaten in einer bestimmten Art und Weise zu formen und in eine Richtung zu lenken. Die emotionale Verinnerlichung bestimmter Werte und gleichzeitig das Ausschalten einer rationalen Hinterfragung der vorgegebenen Normen und Werte waren ihre höchsten Ziele. ${ }^{60}$ So verlangte Hermann Fegelein, zu dieser Zeit Kommandeur des SS-Totenkopf-Reiteregiments 1, am 19. November 1940, dass weltanschauliche Erziehung „immer und überall“ zu erfolgen hätte. ${ }^{61}$ Die Historiker Wegner und Förster relativieren aber in ihren Untersuchungen, dass „weltanschauliche Schulung“ in der Praxis spontan im Stundenplan verschoben, zwischendurch nach Gutdünken abgehalten oder ganz gestrichen wurde. ${ }^{62}$ Noch nach der Niederlage von Stalingrad, Ende Februar 1943, unternahm Berger den Versuch, den Anteil Wochenstunden der ,weltanschaulichen Erziehung“ auf acht zu erhöhen, obwohl offensichtlich war, dass den Truppen an der Front Zeit und Ruhe fehlten, um einen „planmäßigen Schulunterricht“ durchlaufen zu können. ${ }^{63}$ Selbst als der Krieg weit fortgeschritten war, wurden die Kompanieführer der Division „Handschar“ am 2. April 1944 angewiesen, dass weltanschauliche

57 Vgl. Böhler, Gerwarth, The Waffen-SS, S. 11-13.

58 Vgl. Rudolf Brandt, Persönlicher Stab Reichsführer SS an OKW, 13.10.1942, PAAA R100696, Bl. 130288.

59 Vgl. Zaugg, Albanische Muslime, S. 190.

60 Vgl. Leleu, La Waffen-SS, S. 413-417 Vgl. Wegner, Soldaten, S. $168 \mathrm{f}$.

61 1. SS-T.-Reiterregiment, „Dienstanweisung für weltanschauliche Erziehung“, Hermann Fegelein, BArchF RS 4/156, zitiert nach Jürgen Förster, Die weltanschauliche Erziehung in der Waffen-SS. „Kein totes Wissen, sondern lebendiger Nationalsozialismus“, S. 97. Vgl. auch Wegner, Soldaten, S. 191.

62 Vgl. Wegner, Soldaten, S. 167, 193f. Vgl. Förster, Die weltanschauliche Erziehung, S. 97.

63 Chef SS-HA, Durchführungsbestimmungen v. 13.5.1943 zum SS-Befehl v. 24.2. 1943, BArchF: RS 3-9/13, S. 1, zu c: I/1, zitiert nach Wegner, Soldaten, S. 195. 
Erziehung immer dann zu erfolgen habe, „wenn der richtige Augenblick es erfordert oder zwanglose Unterhaltungen die Möglichkeiten geben, in Abendstunden, am Biwakfeuer, bei der Zigarette nach dem Abendessen“. ${ }^{64}$ So ist für diese Division belegt, dass die Abteilung VI noch im Frühjahr 1944 ein Verhältnis von „,60\% weltanschauliche Schulung und 40\% Waffenausbildung“" ankündigte. ${ }^{65}$

64 13. SS-Division, Abt. VI, Anweisung „für den Kompanieführer“, v. 2.4.1944, BArchB: NS 19/ neu 2601, zit. nach Wegner, Soldaten, S. 191. Vgl. Zaugg, Albanische Muslime, S. 190. Siehe zu unkonventionellen Methoden der „weltanschaulichen“ Erziehung, beispielsweise in Form von Gemeinschaftsabenden oder pseudoreligiösen Morgenfeiern, Wegner, Soldaten, S. 191.

65 13. SS-Division, Abt. VI an Kompanieführer, „Weltanschauliche und politische Ausrichtung der Truppe im besonderen Einsatz“, 2.4.1944, BArchB, NS 19/2601, Bl. 229. 